\title{
Kojibiose ameliorates arachidic acid-induced metabolic alterations in hyperglycaemic rats
}

\author{
José Moisés Laparra ${ }^{1 *}$, Marina Díez-Municio ${ }^{2}$, F. Javier Moreno ${ }^{2}$ and Miguel Herrero ${ }^{2}$ \\ ${ }^{1}$ Instituto Universitario de Ingeniería de Alimentos para el Desarrollo (IIAD), Universidad Politécnica de Valencia, Valencia \\ 46022, Spain \\ ${ }^{2}$ Instituto de Investigación en Ciencias de la Alimentación, CIAL (CSIC-UAM), CEI (UAM+CSIC), Madrid 28049, Spain \\ (Submitted 4 May 2015 - Final revision received 8 July 2015 - Accepted 21 July 2015 - First published online 7 September 2015)
}

\begin{abstract}
Herein we hypothesise the positive effects of kojibiose (KJ), a prebiotic disaccharide, selected for reducing hepatic expression of inflammatory markers in vivo that could modulate the severity of saturated arachidic acid (ARa)-induced liver dysfunction in hyperglycaemic rats. Animals were fed daily (20 d) with ARa $(0.3 \mathrm{mg})$ together or not with $\mathrm{KJ}(22 \mathrm{mg}$ approximately $0.5 \%$, w/w diet). Glucose, total TAG and cholesterol contents and the phospholipid profile were determined in serum samples. Liver sections were collected for the expression (mRNA) of enzymes and innate biomarkers, and intrahepatic macrophage and T-cell populations were analysed by flow cytometry. ARa administration increased the proportion of liver to body weight that was associated with an increased (by $11 \%$ ) intrahepatic macrophage population. These effects were ameliorated when feeding with KJ, which also normalised the plasmatic levels of TAG and $N$-acyl-phosphatidylethenolamine in response to tissue damage. These results indicate that daily supplementation of KJ significantly improves the severity of ARa-induced hepatic alterations.
\end{abstract}

Key words: Kojibiose: Arachidic acid: Liver inflammation: Steatosis: Hyperglycaemia

Prebiotics are dietary substances (mostly consisting of oligosaccharides and NSP, poorly digested by human enzymes) that are prone to be fermented by beneficial gut microbiota involving concomitant health implications. Kojibiose (KJ, 2-O- $\alpha$ D-glucopyranosyl- $\alpha$-D-glucopyranose) is a naturally occurring carbohydrate that can be found in a variety of foodstuffs and beverages, such as sake and koji extracts, beer, honey or starch hydrolysate. However, $\mathrm{KJ}$ is naturally present at low levels, making its isolation from natural sources difficult at high scale and demanding an efficient biotechnological synthesis process $^{(1)}$. Interestingly, $\mathrm{KJ}$ has been reported to be an excellent candidate as a prebiotic ingredient according to its in vitro selectivity of microbial fermentation using individual bacteria from Bifidobacterium, Streptococcus, Lactobacillus or Eubacterium genera ${ }^{(2,3)}$, as well as with cultures of mixed human faecal bacteria ${ }^{(4)}$.

The European Food Safety Authority has recently approved a health claim linking non-digestible carbohydrates (i.e. fructooligosaccharides (FOS)) and a reduction of postprandial glycaemic (and insulinaemic) responses ${ }^{(5)}$. Notwithstanding, opposite results have been obtained from type 2 diabetic (T2D) patients about the regulatory effect of prebiotic on glucose homoeostasis ${ }^{(6)}$. Besides, the reported prebiotic-mediated effects on circulating blood levels in hyperlipidaemic human subjects appear variable ${ }^{(7)}$.

Prior research has largely focused on total energy intake and consumption, with a continuous positive balance promoting diseases such as obesity, fatty liver and finally nonalcoholic fatty liver disease/nonalcoholic steatohepatitis (NAFLD/NASH) and/or the metabolic syndrome. NAFLD incurs a high risk for the development of $\mathrm{T} 2 \mathrm{D}$ and other major features of the metabolic syndrome; thus, plasma glucose and TAG, among others, constitute risk factors of NAFLD. Recent data suggest that the composition of the food, irrespective of energy count, and its influence on and interaction with the gut microbiota, and finally their crosstalk with the host's intestinal immune system, may be even more important determinants of intestinal and liver-related disorders ${ }^{(7,8)}$. Experimental data and incipient human studies indicate the positive effects of prebiotics in improving the regulation of glucose homoeostasis, in addition to influencing the immune function both directly through their interaction with innate mediators and/or by modifying the gut microbiota composition ${ }^{(9,10)}$.

The liver acts as a central organ for the turnover and transformation of nutrients and metabolites that pass through it from

Abbreviations: $A N G P 4$, angiopoietin-like protein 4; ARa, arachidic acid; KJ, kojibiose; LPC, lysophosphatidylcholine; PL, phospholipid; STZ, streptozotocin; TLR4, toll-like receptor-4.

* Corresponding author: J. M. Laparra, fax +34 963877956, email moilallo@upvnet.upv.es 
the intestine. In addition, the intestine also supplies regulatory signals of inflammatory processes that stem from the intestinal mucosa and immune system, highlighting the importance of the so-called gut-liver axis in the development of metabolic and liver-related diseases. Experimental data from murine models support the potential beneficial influence of prebiotics on liver function, contributing to normalising the hepatic $P P A R-\gamma$ mRNA expression and the production of proinflammatory hepcidin peptide $^{(11)}$. Fermentation of prebiotics was also positively associated with decreased glucose-induced TNF- $\alpha$ and IL- 6 levels in human subjects ${ }^{(12)}$. Furthermore, prebiotic structures can potentially interact with innate mediators through the toll-like receptor (TLR)-4/NF- $\kappa \mathrm{B}$ signalling pathway ${ }^{(13,14)}$, thus promoting positive effects such as reducing TAG accumulation and the endogenous synthesis of cholesterol, favouring liver physiology $^{(7,15)}$. Alterations in the phospholipid (PL) profile have also been reported in diabetic patients ${ }^{(16)}$, pointing to these as new players with a key role in metabolic diseases, but there is no evidence of the influence of prebiotic feeding on either humans or animal models.

Several attempts have been made to develop animal models approaching major pathophysiological manifestations of diet-induced metabolic disorders such as obesity and insulin resistance $^{(17)}$. Overall, there is still an open debate about the definition of a low- ( $<10 \%$ of energy) or high-fat ( $>30-50 \%$ of energy) diet, and a wide variety of diets are used in animal studies. Rodents are less sensitive to the development of metabolic disorders, thus requiring greater fat intake than do humans to induce metabolic abnormalities. Additionally, there is a lack of data on how and which prebiotics could modulate metabolic derangement and finally liver dysfunction. In this context, treatment of animals with a low dose of streptozotocin (STZ) resulted in hyperglycaemia in animals without requiring exogenous insulin administration ${ }^{(18,19)}$. Thus, this model together with the concurrent administration of SFA approaches early stages of fat-induced liver alterations that ultimately could promote liver diseases. Undoubtedly, further refinement of the available animal models is desirable, but it is considered appropriate to explore initial pathogenic mechanisms and potential pharmaceutical or nutritional interventions at early stages of liver dysfunction.

KJ has demonstrated advantageous features ameliorating inflammation-mediated perturbations in the liver in comparison with 4-galactosyl-kojibiose ( $O$ - $\beta$-D-galactopyranosyl-(1,4)- $O$ ( $\alpha$-D-glucopyranosyl-(1,2))- $\alpha$-D-glucopyranose) and galactooligosaccharides derived from lactulose ${ }^{(20)}$. Thus, the objective of this work was to study the influence of KJ-based feeding on arachidic acid (ARa)-induced liver injury in a hyperglycaemic model of young rats.

\section{Methods}

\section{Synthesis and purification of kojibiose}

$\mathrm{KJ}(65 \%$ purity on DM) was obtained from the hydrolytic action of a Kluyveromyces lactis $\beta$-galactosidase on $4^{\prime}$-galactosylkojibiose and purified by using a Saccharomyces cerevisiae yeast treatment as has previously been shown. The second most important compound was leucrose (19\%) followed by $8 \%$ of yeast metabolites produced during yeast fermentation (mainly minor amounts of polyalcohols and/or organic acids). Microbiological assays demonstrated that the microbial load (yeast and moulds, total and sporulated aerobic bacteria, enterobacteria) was, in all cases, lower than $3 \times 101$ colony forming unit/g $\mathrm{g}^{(1)}$.

\section{Animals}

For the studies, female Wistar albino rats, aged 3 weeks with an average weight of 61.4 (SD 5.6) g, were obtained from the University of Valencia Animal Service. Animal experiments were carried out in strict accordance with the recommendations in the Guide for the Care and Use of Laboratory Animals of University of Valencia (SCSIE, University of Valencia, Spain) and the protocol (2013/007/UVEG005 type 2) was approved by its Ethics Committee.

\section{Experimental design}

Animals were randomly distributed into four different groups ( $n$ 7/group): (1) controls received a standard AIN-93G diet, and three groups were treated with a low dose of STZ (two doses of $25 \mathrm{mg} / \mathrm{kg}$ per $\mathrm{d}$ with a time lapse of $24 \mathrm{~h})^{(17)}$ and subjected to different treatments; ( 2 fed the standard diet; (3 administered ARa (20 : 0) - 0.3 mg daily for $20 \mathrm{~d}$; (4) administered ARa $0.3 \mathrm{mg}$ daily for $20 \mathrm{~d}$ together with $\mathrm{KJ}(0.5 \%$, w/w daily consumption of diet) during the period of treatment ${ }^{(21)}$. The rats were maintained in an environment of controlled temperature $\left(21-23^{\circ} \mathrm{C}\right)$, humidity $(55 \%)$ and $12 \mathrm{~h}$ light $-12 \mathrm{~h}$ dark cycle, with food and mineral-free water provided ad libitum. Weight gain and food intake were measured every $2 \mathrm{~d}$ and monitored throughout the experiment. Food uptake per body weight and weight gain per food uptake were determined in each group. After treatment, rats were anaesthetised (isofluran) and killed by exsanguination (heart puncture).

\section{Blood collection and morphometric evaluation of the liver}

Whole blood samples were preserved in EDTA-treated tubes to prevent coagulation (at room temperature) for haematological analyses. Total concentration ( $\mathrm{mmol} / \mathrm{l})$ of glucose (Química Analítica Aplicada SA), TAG and cholesterol (Química Analítica Aplicada SA) was quantified using commercial kits commonly used for clinical diagnosis. Analytical interferences were discarded by confirmation of the standard compound provided in the commercial kit.

Complete livers were extracted, rinsed with Krebs' buffer and weighed. Thereafter, sections (approximately $100 \mathrm{mg}$ ) of the liver were immersed in RNAlater buffer (Qiagen) and snapfrozen in liquid $\mathrm{N}_{2}$ for gene expression analyses. Samples from central lobules $(0.51$ (SD 0.04) g) were immersed in a trypsin solution for $30 \mathrm{~min}$ before homogenisation and immune cell analysis by flow cytometry (Coulter). The following fluorochrome-conjugated antibodies were used: anti-rat CD45, CD4, CD8 and Cd11b/c (eBioscience); fluorescence was analysed by flow cytometry (EPICS XL-MCL). Samples were 
prepared with the Immunoprep kit (Beckman Coulter) according to the manufacturer's instructions and further analysed on an FACSCanto System (BD Biosciences).

\section{Real-time RT-quantitative PCR}

Total RNA was extracted from liver tissue samples using an RNeasy mini kit (Qiagen) following the protocol provided by the manufacturer. One microgram of total RNA was converted to double-stranded complementary DNA (cDNA) using AMV RT (Promega). PCR was performed with primers designed for the following Rattus norvegicus genes: angiopoietin-like protein 4 (ANGP4) (forward 5'-CTT TTT ACG CTG CTG CCG TT-3', reverse 5'-AAC CCA AGA CCT TGA CCG TG-3'); TLR4 (forward 5'-TTC TCA CGG CCT CCG CTG GT-3', reverse 5'-AAG GGA GGC TAA CCG GAA AGG GA-3'); LDL receptor ( $L D L r$ ) (forward 5'-GGA CCC GTG ATC CTG TGT TT-3', reverse 5'-AAA GAA AAC CAC TCG GGG CT-3'); PPAR (forward 5'-TGA TCC TAC GGC CAG ACA GA-3', reverse 5'-GGG AGG TTG TCC CTG GAA TG-3') and $\beta$-actin (forward 5'-CTC TTC CAG CCT TCC TTC CT- $3^{\prime}$, reverse $5^{\prime}$-TAG AGC CAC CAA TCC ACA CA-3'); the latter was used as a housekeeping gene. Samples of each animal tissue were measured in duplicate, and gene expression was expressed as fold-change. The PCR mix $(25 \mu \mathrm{l}$ reaction volume) in triplicate consisted of $7.5 \mu \mathrm{l}$ SYBR Green I Master mix (Roche), $1.25 \mu \mathrm{mol} / 1$ primers and $2.5 \mu \mathrm{l}$ cDNA. PCR reactions were performed in a LightCycler ${ }^{\circledR} 480$ (Roche) system under the following conditions: one cycle at $95^{\circ} \mathrm{C}$ for $5 \mathrm{~min}$, and forty cycles at $60^{\circ} \mathrm{C}$ for $20 \mathrm{~s}$ and at $72^{\circ} \mathrm{C}$ for $45 \mathrm{~s}$. Data were analysed using the $2^{-\Delta C_{t}}$ method, being normalised against $\beta$-actin and expressed as fold-change.

\section{Serum arachidic acid quantification and phospholipids profile}

Blood samples $(0 \cdot 3 \mathrm{ml})$ were centrifuged $(3000 \boldsymbol{g}$ for $10 \mathrm{~min}$ at $4^{\circ} \mathrm{C}$ ) (Jouan) and the supernatant mixed with $700 \mu \mathrm{l}$ formic acid $(0 \cdot 1 \%, \mathrm{v} / \mathrm{v})$ in acetonitrile. The mixtures were loaded onto HybridSPE $^{\circledR}$-PL cartridges (Cat No. 55269-U; Supelco) to purify the PL fraction ${ }^{(22)}$.

The analysis was performed on an Agilent 1260 HPLC system (Agilent) connected on line to a QqTOF MS (5600 TripleTOF; AB Sciex) equipped with an electrospray interface. The column used in these analyses was a C18 $1.7 \mu \mathrm{m}$ particle, $2.1 \times 50 \mathrm{~mm}$ (Waters). The mobile phases consisted of deionised water, 18 $\mathrm{M} \Omega \mathrm{cm}(\mathrm{A})$ and acetonitrile (B), both containing $0 \cdot 1 \%(\mathrm{v} / \mathrm{v})$ formic acid. Aliquots $(5 \mu \mathrm{l})$ of the PL purified fractions were injected in each cycle and the analysis was performed with the following gradient $(0.8 \mathrm{ml} / \mathrm{min}): 20 \% \mathrm{~B}$ for $2 \mathrm{~min}$ to $100 \% \mathrm{~B}$ in $16 \mathrm{~min}$, held at $100 \%$ for $7 \mathrm{~min}$. Two independent samples from each animal were analysed. The QqTOF MS instrument was operated under the following conditions: nebulisation and solvation pressure, $50 \mathrm{psi}$; curtain gas pressure, $30 \mathrm{psi}$; temperature, $450^{\circ} \mathrm{C}$; ion-spray voltage floating, $3500 \mathrm{~V}$; de-clustering potential, $110 \mathrm{~V}$; and energy collision, $60 \mathrm{~V}$. Serum ARa concentrations were quantified using appropriate standard curves. Areas corresponding to the total ion current were used to compare the PL profile.

\section{Analysis of SCFA and lactic acid}

Aliquots $(0.17$ (sD 0.04) g) of colon samples were kept in $0.2 \mathrm{ml}$ of $2 \mathrm{~N}-\mathrm{H}_{2} \mathrm{SO}_{4}$. The samples were homogenised ( $1 \mathrm{~min}$ ) using a TissueRuptor (Qiagen) and vortexed for $30 \mathrm{~s}$. Afterwards, the mixtures were centrifuged (10000 g, $10 \mathrm{~min}$ ) and the supernatant was collected and diluted in deionised water prior to filtration ( $0.45 \mu \mathrm{m}$, MillexGN; Millipore).

The quantification of organic acids was carried out on a 1200 Agilent HPLC system equipped with a multisolvent pump and a multivariable wavelength absorbance detector set at $214 \mathrm{~nm}$ (1200 Series; Agilent Technologies). The separation was performed on a BioBasis C18 column $(250 \times 4.6 \mathrm{~mm})$ (Thermo). The elution was performed using $1 \%$ acetronitrile in $20 \mathrm{~mm}-$ phosphate buffer adjusted to $\mathrm{pH} 2 \cdot 20$ with phosphoric acid (A) and water-acetronitrile $(80: 20, \mathrm{v} / \mathrm{v}, \mathrm{B})$ according to the following gradient: $0 \mathrm{~min}, 0 \% \mathrm{~B} ; 5 \mathrm{~min}, 0 \% \mathrm{~B} ; 12 \mathrm{~min}, 10 \% \mathrm{~B}$; $19 \min , 10 \% \mathrm{~B}$.

\section{Statistical analysis}

Statistical analyses were performed using SPSS version15 software (SPSS Inc.). Differences between the different groups of treatments were established with the Student's $t$ test. One-way ANOVA was used to compare the influence of feeding $\mathrm{KJ}$ to the different treated groups of animals. Individual means were tested using pairwise comparison with Tukey's multiple comparison tests when effects were significant. Statistical significance was established at $P<0.05$ for all comparisons.

\section{Results \\ Changes in body and liver weight}

There were no significant differences in daily food (energy) intake between the different groups of treatment $(71.5 \mathrm{~kJ} / \mathrm{d}$ $(17 \cdot 1 \mathrm{kcal} / \mathrm{d}), 17 \cdot 7 \%$ protein, $60.1 \%$ carbohydrate, $7 \cdot 2 \%$ fat $)$ over the study period. The lower body weight of animals administered $\mathrm{ARa} / \mathrm{KJ}$ (approximately $20 \%$ ) in relation to those administered only ARa (Fig. 1(A)) suggests that energy intake is not the only determinant in the development of the disease. Morphometric comparison of the proportion of liver to body weight revealed significant $(P<0.05)$ increases in the liver weight of all hyperglycaemic animals relative to untreated controls (Fig. 1(B)). Notably, animals administered ARa/KJ showed significantly lower $(P=0.01)$ proportions of liver to body weight than did animals fed ARa alone.

\section{Biochemical parameters}

Serum concentrations of biochemical parameters of metabolic relevance are shown in Table 1. Animals treated with STZ showed a 2 -fold increase in serum glucose and TAG and a 4-fold increase in cholesterol concentration relative to the untreated controls. The administration of ARa alone or together with KJ affected the mean serum glucose concentrations aggravating hyperglycaemia. The administration of ARa was significantly associated with a $2 \cdot 5$-fold increase in TAG levels 
compared with animals treated with STZ, but not total cholesterol. In addition, the concurrent administration of ARa together with $\mathrm{KJ}$ is associated with decreased serum TAG concentration in relation to animals administered ARa alone.

\section{Expression of hepatic biomarkers}

Changes in hepatic expression levels of biomarkers of lipid metabolism and innate immune mediators are shown in Fig. 2.
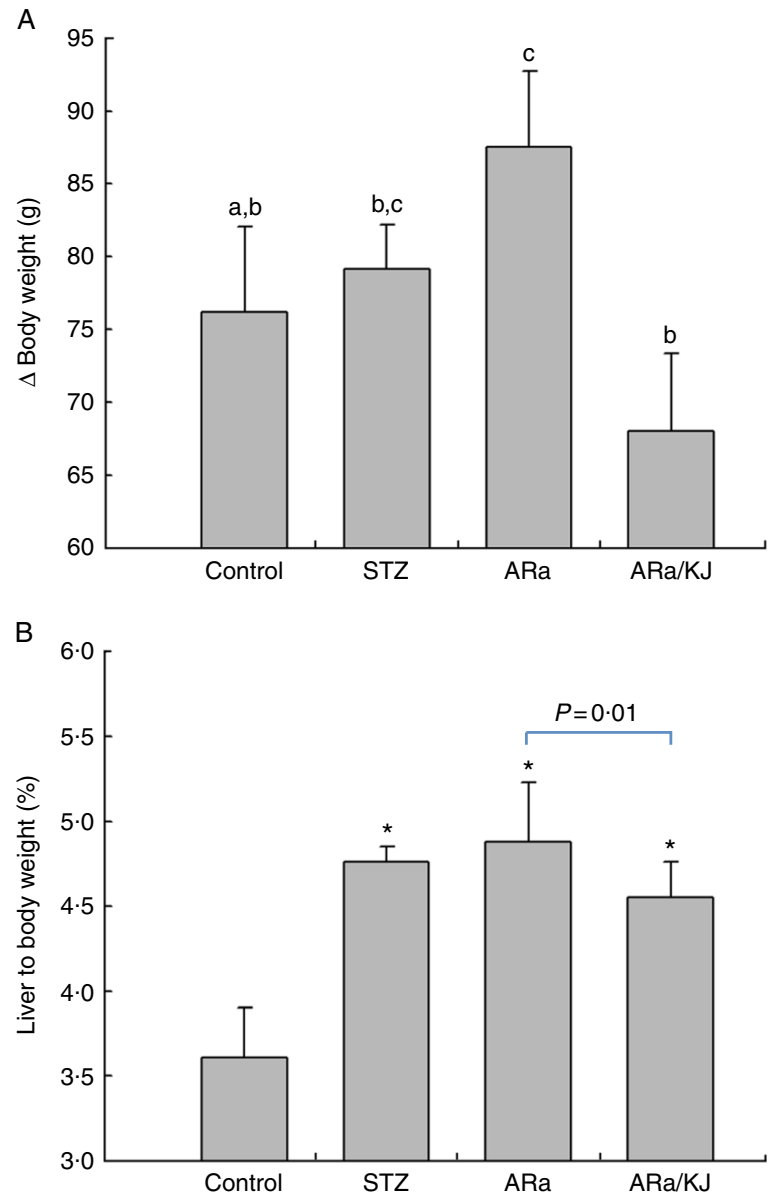

Fig. 1. Changes in body weight (A) and the relative liver to body weight (B) in untreated controls and hyperglycaemic animals administered saturated arachidic acid (ARa) alone or together with kojibiose (ARa/KJ). Values are means $(n 7)$, and standard deviations represented by vertical bars. ${ }^{a, b, c}$ Mean values with unlike letters were significantly different $(P<0.05)$ between the different groups of treatment. * Mean value was significantly different from of the control group $(P<0.05)$, STZ. streptozotocin.
Treatment of animals with STZ increased mRNA transcripts of all targeted genes compared with controls. Additionally, animals fed ARa showed significantly increased expression levels of both $A N G P 4$ and $L D L r$, reflecting the physiological response to inhibit fat uptake and favour the reverse transport of cholesterol to the liver. KJ did not exert any additional effect when it was fed to animals administered ARa. However, animals fed ARa together with KJ showed significantly increased expression values of PPAR $\alpha$ in comparison with those fed only ARa, suggesting an increased fatty acid metabolism. Animals administered ARa showed higher transcripts of TLR4, and those values were not affected by the administration of $\mathrm{KJ}$.

\section{Intrahepatic lymphocyte and macrophage population}

The leucocyte population was identified with the marker CD45, and further phenotyping analyses of lymphocyte subsets $(\mathrm{CD} 4+, \mathrm{CD} 8+)$ and macrophage (CD11b/c+) populations were conducted on this population (Fig. 3). Treating animals with STZ only significantly increased the numbers of infiltrated CD4+ cells. Administration of ARa did not cause further significant increases in either the CD4+ or the CD8+ population, although intrahepatic CD11b/c+ cells increased by $31.5 \%$. Feeding KJ to animals administered ARa had positive effects in reducing CD11b/c+ cells to counts similar to those found in STZ-treated animals.

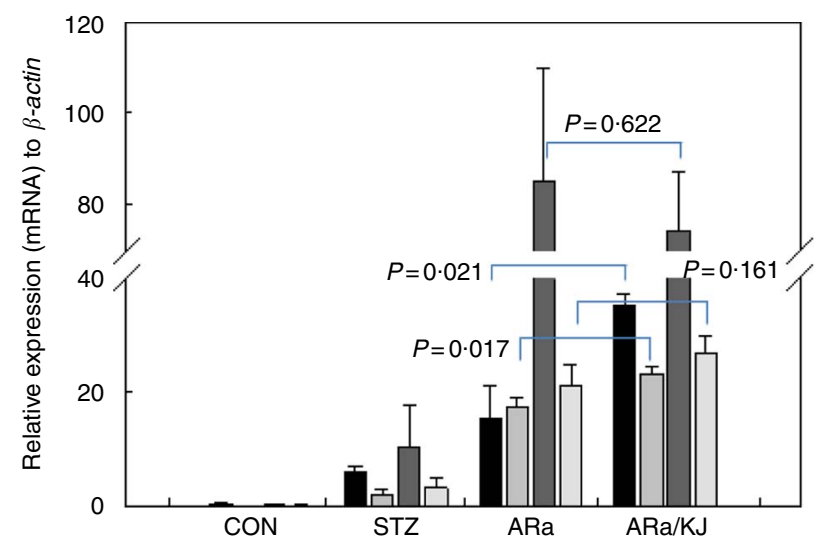

Fig. 2. Hepatic expression of hepatic biomarkers in untreated controls (CON) and hyperglycaemic animals administered saturated arachidic acid (ARa) alone or together with kojibiose $(\mathrm{ARa} / \mathrm{KJ})$. Values are means $(n 7)$, and standard deviations represented by vertical bars. $\square$, PPARa; $\square$, angiopoietinlike protein 4 (ANGP4); $\square$, LDL receptor $(L D L r)$; $\square$, toll-like receptor 4 (TLR4); STZ, streptozotocin.

Table 1. Total glucose (GLUC), TAG and cholesterol (CHOL) concentrations ( $\mathrm{mmol} / \mathrm{l})$ in untreated controls and hyperglycaemic animals administered saturated arachidic acid (ARa) alone or together with kojibiose (ARa/KJ)

(Mean values and standard deviations; $n$ )

\begin{tabular}{|c|c|c|c|c|c|c|c|c|}
\hline \multirow[b]{2}{*}{ Treatment } & \multicolumn{2}{|c|}{ GLUC (mmol/l) } & \multicolumn{2}{|c|}{ TAG $(\mathrm{mmol} / \mathrm{l})$} & \multicolumn{2}{|c|}{$\mathrm{CHOL}(\mathrm{mmol} / \mathrm{l})$} & \multicolumn{2}{|c|}{$\mathrm{ARa}(\mathrm{mmol} / \mathrm{l})$} \\
\hline & Mean & SD & Mean & SD & Mean & SD & Mean & SD \\
\hline Control & $121 \cdot 2^{a}$ & $23 \cdot 3$ & $89 \cdot 9^{a}$ & 7.4 & $100 \cdot 3^{a}$ & $18 \cdot 2$ & $8 \cdot 1^{\mathrm{a}}$ & 0.2 \\
\hline $\mathrm{STZ}$ & $213 \cdot 5^{a, b}$ & $70 \cdot 7$ & $224.5^{\mathrm{b}}$ & 32.4 & $430 \cdot 0^{\mathrm{b}}$ & $22 \cdot 8$ & $10 \cdot 7^{b}$ & 1.2 \\
\hline ARa & $316 \cdot 2^{b, c}$ & $74 \cdot 1$ & $561 \cdot 8^{\mathrm{C}}$ & $43 \cdot 7$ & $454 \cdot 2^{\mathrm{b}}$ & 70.6 & $13 \cdot 9^{c}$ & 0.6 \\
\hline ARa/KJ & $372.1^{c}$ & 39.5 & $264.7^{\mathrm{b}}$ & 19.8 & $417 \cdot 8^{\mathrm{b}}$ & 45.1 & $9 \cdot 7^{\mathrm{a}, \mathrm{b}}$ & 1.0 \\
\hline
\end{tabular}

STZ, streptozotocin.

a,b,c Mean values within a row with unlike superscript letters were significantly different $(P<0.05)$ for each parameter between the different groups of treatment. 


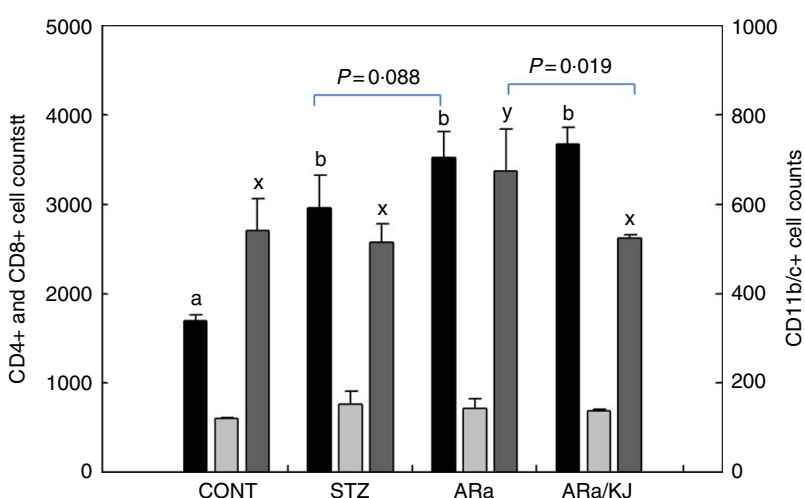

Fig. 3. Phenotyping of intrahepatic lymphocytes (CD4+ and CD8+) and macrophage $(\mathrm{CD} 11 \mathrm{~b} / \mathrm{c}+)$ population in untreated controls $(C O N)$ and hyperglycaemic animals administered saturated arachidic acid (ARa) alone or together with kojibiose (ARa/KJ). Values are means $(n 7)$, and standard deviations represented by vertical bars. ${ }^{a, x, b, y}$ Mean values with unlike letters were significantly different $(P<0.05)$ for each population between the different groups of treatment. $\square, \mathrm{CD} 4+; \square, \mathrm{CD} 8+, \square, \mathrm{CD} 11 \mathrm{~b} / \mathrm{c}+$.

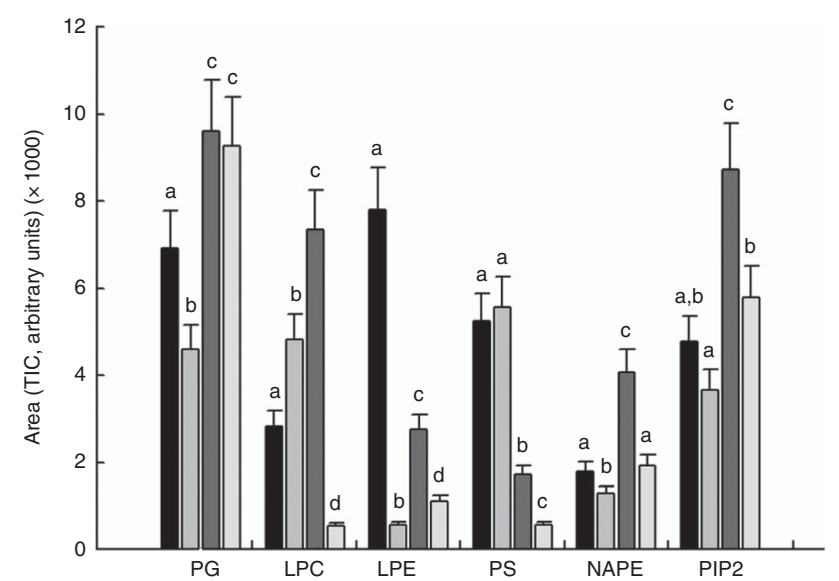

Fig. 4. Plasmatic phospholipid profile in untreated controls and hyperglycaemic animals administered saturated arachidic acid (ARa) alone or together with kojibiose (ARa/KJ). Values are means $(n 7)$, and standard deviations represented by vertical bars. ${ }^{a, b, c, d}$ Mean values with unlike letters were significantly different $(P<0.05)$ for each compound between the different groups of treatment. TIC, total ion current; PG, phosphatidylglycerol; LPC, lysophosphatidylcholine (20:2); LPE, lysophosphatidylethanolamine (18:1); PS, phosphatidylserine; NAPE, $\mathrm{N}$-acylphosphatidylethenolamine (20:2); PIP2, phosphatidylinositol diphosphate. , Control; $\square$, STZ; $\square$, ARa; $\square$, ARa/KJ.

\section{Phospholipid profile}

All PL identified in aliquots of plasma were identified using high-resolution MS after liquid chromatography (LC-QqTOF MS). The groups of animals treated with STZ that were not fed either with ARa or KJ showed higher lysophosphatidylcholine (LPC) and non-altered concentration of phosphatidylserine (PS), whereas plasma levels of all other identified PL were decreased in comparison with control animals (Fig. 4). In animals fed ARa, decreased levels of PS were detected, whereas all other identified PL were increased. Feeding KJ to animals decreased the concentrations of lysophosphatidylethanolamine (LPE), $N$-acyl-phosphatidylethenolamine (NAPE) and phosphatidylinositol diphosphate (PIP2), approximating these values to those quantified for animals treated with STZ, but not fed ARa. Additionally, the group of animals administered ARa together with KJ showed reduced LPC and PS values than did animals fed only ARa.

\section{Quantification of SCFA and lactic acid}

The concentrations of organic acids quantified in colon contents of animals from the different groups of treatment are shown in Table 2. Significantly lower concentrations of formic acid were detected in all STZ-treated animals compared with control animals, and were slightly increased by the administration of $\mathrm{ARa}$ and not modified despite the sequential administration of KJ. Administration of STZ to animals caused significant increases in acetic and D/L-lactic acid concentrations, which increased even more in ARa-fed animals. Feeding KJ to animals only reduced the concentration of D/L-lactic acid significantly. An opposite pattern was observed for propionic acid that appeared at lowest concentration in ARa-fed animals and normalised to the values found in the STZ group by the administration of KJ. $i$-Butyric acid concentrations only significantly increased in the ARa group.

\section{Discussion}

This study reports findings to expand the positive effects of feeding prebiotics, particularly the influence of $\mathrm{KJ}$ on the saturated ARa-induced alterations in an experimental hyperglycaemic animal model. ARa was chosen because of its central

Table 2. SCFA and lactic acid production in colon contents $(\mu \mathrm{g} / \mathrm{g})$ of untreated controls and hyperglycaemic animals administered saturated arachidic acid (ARa) alone or together with kojibiose (ARa/KJ)

(Mean values and standard deviations; $n$ 7)

\begin{tabular}{|c|c|c|c|c|c|c|c|c|}
\hline \multirow[b]{2}{*}{ Organic acids } & \multicolumn{2}{|c|}{ Control } & \multicolumn{2}{|c|}{ STZ } & \multicolumn{2}{|c|}{ ARa } & \multicolumn{2}{|c|}{ ARa/KJ } \\
\hline & Mean & SD & Mean & $\mathrm{SD}$ & Mean & $\mathrm{SD}$ & Mean & SD \\
\hline Formic & $9.93^{\mathrm{a}}$ & $1 \cdot 15$ & $3.06^{\mathrm{b}}$ & 0.31 & $5 \cdot 23^{c}$ & 1.33 & $5 \cdot 47^{\mathrm{c}}$ & $1 \cdot 19$ \\
\hline Acetic & $1.63^{\mathrm{a}}$ & 0.19 & $2 \cdot 79^{\mathrm{b}}$ & 0.28 & $4.60^{c}$ & 1.36 & $3 \cdot 58^{\mathrm{b}, \mathrm{c}}$ & 1.20 \\
\hline Propionic & $0.63^{\mathrm{a}}$ & 0.07 & $0.29^{\mathrm{b}}$ & 0.10 & $0.14^{\mathrm{C}}$ & 0.02 & $0.39^{b}$ & 0.04 \\
\hline D/L-Lactic & $0 \cdot 24^{a}$ & 0.03 & $1.36^{\mathrm{b}}$ & 0.18 & $9.09^{c}$ & 1.61 & $1.36^{\mathrm{b}}$ & 0.18 \\
\hline i-Butyric & $0.28^{a}$ & 0.03 & $0.29^{\mathrm{a}}$ & 0.05 & $0.81^{\mathrm{b}}$ & 0.26 & $0.33^{\mathrm{a}}$ & 0.19 \\
\hline
\end{tabular}

STZ, streptozotocin.

a,b,c Mean values within a row with unlike superscript letters were significantly different $(P<0.05)$ for each parameter between the different groups of treatment. 
role in inflammation related to injury and many diseased states and as a lipid second messenger involved in the regulation of signalling enzymes, such as phospholipase C (PLC)- $\gamma$, PLC- $\delta$, and protein kinase $\mathrm{C}-\alpha,-\beta$, and $-\gamma$ isoforms ${ }^{(23)}$. On the basis of the identification of beneficial prebiotics, even without a difficult-toachieve massive energy reduction, a better understanding and the use of beneficial prebiotics can greatly impact the risk of developing liver-related disorders. As shown, consumption of $\mathrm{KJ}$ significantly decreased the plasmatic levels of ARa, and probably a lower production of hepatotoxic products, which in turn leads to a low-grade inflammation state and liver dysfunction. Feeding $\mathrm{KJ}$ increased the numbers of Bifidobacterium spp. (by 12\%), Bacteroides spp. (by 2\%) and Enterobacteriaceae (by 4\%) relative to control animals (data not shown).

The animal model used in the present study approaches early metabolic alterations that, in patients, ultimately can lead to liver dysfunction and steatosis with inflammation. Notably, there is a quantified increase in macrophage population in the liver as well as plasma levels of NAPE, an important intermediary in the biosynthesis of endocannabioid (eCB) agonists such as anandamide ${ }^{(24)}$. These results are concordant with previous data in which the increased anandamide concentrations were associated with hepatic lipogenesis in high-fat-fed mice $^{(25)}$. Taking these data together, a role for the eCB system in the development of obesity could be suggested, at least in rodents, probably with an immunomodulatory role in the activation of inflammasome in macrophages ${ }^{(26)}$. The increased T-cell CD4+ population quantified in animals fed ARa is concordant with the CD28-dependent signalling initiated by the conversion of PIP2 into phosphatidylinositol (3,4,5)-trisphosphate (PIP3) ${ }^{(27)}$ that can explain the increased PIP2 concentration quantified (Fig. 4). However, this study shows that the period of treatment is not enough to reproduce the T-cell recruitment involved, effector CD8+ T-cells that are associated with late steatosis with inflammation (NASH) ${ }^{(28)}$.

From these data, one can derive an important role for prebiotics in influencing the lipid metabolism, affecting the central regulatory mechanisms to control fatty acid oxidation (i.e. $P P A R \alpha$ ) and affect fat absorption and turnover directly in the gut (i.e. ANGP4). The decreased TAG concentrations in animals fed $\mathrm{KJ}$ associated with increased transcripts of $P P A R \alpha$ (Table 1 and Fig. 2) are in good accordance with the proven effect of FOS in reducing hepatic TAG accumulation through a PPAR $\alpha$ stimulation of fatty acid oxidation ${ }^{(8)}$. Overall, detailed information on metabolism in mitochondria, a vital organelle for fatty acid oxidation, is especially lacking and would be important to further evaluate the influence of prebiotics on the regulation of liver-related diseases. The administration of $\mathrm{KJ}$ caused a significant increase in ANGP4 mRNA expression in comparison with those values determined in ARa-fed animals (Fig. 2), thus suggesting that it mainly affected fatty acid metabolism, preventing macrophage activation in mesenteric lymph nodes $^{(29)}$. This could help to explain the decreased proportion of macrophages in the liver in animals fed ARa/KJ (Fig. 4). The observation that $\mathrm{ARa} / \mathrm{KJ}$-fed animals showed decreased TAG concentrations (Table 1) allows hypothesising a posttranscriptional control of $A N G P 4$. This is in agreement with the reported proteolytic cleavage of $A N G P 4$ at the linker region, releasing $n \mathrm{ANGP} 4$ and the monomeric C-terminal portion of $A N G P 4$, which exert different biological functions ${ }^{(30)}$. A differential PPAR $(-\alpha,-\gamma$ and $-\delta)$ mRNA expression has been seen to be modulated by long-chain fatty acids (LCFA), which led to the suggestion that these compounds could act as ligand but not as regulators of these receptors ${ }^{(31)}$. Thus, the decreased plasmatic concentration of ARa in KJ-fed animals indicates the positive influence of $\mathrm{KJ}$ in limiting the absorption of ARa that could constitute an additional factor contributing to the LCFAmediated regulation of $P P A R$, as previously indicated ${ }^{(31)}$. As a consequence, a decreased production of the biologically active $\mathrm{n} / \mathrm{c}-\mathrm{ANGP} 4$ monomers relative to the ARa group could reduce the mobilisation of fatty acids from storage tissues as well as macrophage activation ${ }^{(32)}$. These changes are in agreement with the decreased CD11b/c+ cell proportion infiltrated into the liver of the $\mathrm{ARa} / \mathrm{KJ}$ group relative to ARa-fed animals.

The previously reported ANGP4-mediated inhibition of lipoprotein lipase activity, leading to hypertriglyceridaemia ${ }^{(33)}$ and enhanced uptake of TAG promoted by interaction of apoE with the $L D L r$, is in accordance with the significantly increased $L D L r$ mRNA expression levels in animals fed ARa (Fig. 2). Feeding KJ to animals that received ARa favoured a positive effect, reducing the plasmatic TAG and ARa concentrations (Table 1); however, administration of $\mathrm{KJ}$ failed to positively decrease cholesterol concentrations, supporting the absence of statistically significant changes in $L D L r$ mRNA expression (Fig. 2). The increased accumulation of TAG and their subsequent oxidation could favour inflammatory response(s) through interaction with $T L R 4^{(34)}$, supported by an increased TLR4 mRNA expression level in animals fed ARa. In this scenario, the administration of $\mathrm{KJ}$ failed to decrease the hepatic TLR4 expression levels of ARa/KJfed animals (Fig. 2). Metabolic effects derived from the production of SCFA from prebiotic consumption could also be expected in relation to lipogenesis and energy homoeostasis ${ }^{(34)}$. For example, there has been reported a protective role for these SCFA against diet-induced obesity due to their regulatory effects on the production of gut hormones such as glucagon-like peptide-1 (GLP-1) and peptide tyrosine tyrosine (PYY) through their interaction with the free fatty acid receptor 2 (FFAR2; also known as GPR43) ${ }^{(35,36)}$. Animals fed KJ showed concentrations of both propionate $(0.18 \mathrm{~mm})$ and butyrate $(0.13 \mathrm{~mm})$ that were much lower than those favouring the secretion of GLP-1 and PYY production. Unfortunately, the experimental design of this study does not allow to either directly associate or negate the potential influence of SCFA on the liver inflammatory biomarkers.

Changes in lipid metabolism have been further characterised in relation to the PL profile, which are building blocks in biological membranes and potent mediators in cell homoeostasis and inflammation, and in macrophage and neutrophil chemotaxis. Recent metabolome-based studies have also associated alterations in immunomodulatory lipid components such as PL levels with obesity and $\mathrm{T} 2 \mathrm{D}^{(16)}$. These alterations are observed in hyperglycaemic animals that showed increased plasmatic levels of LPC and decreased LPE (Fig. 4). Time-course monitoring studies for 3 weeks revealed different patterns for the LPC species, showing early (after 1 week) increases for circulating LPC (20:0) and LPC (20:4). However, decreased levels for LPC (20:1) were reported during the first 3 weeks of 
monitoring, whereas LPC (20:5) levels decreased during the 1st week of treatment, but increased during the next 2 weeks ${ }^{(35,36)}$. A similar pattern with increased circulating levels of LPC (20:2) with low saturations was detected in ARa-fed animals (Fig. 4). Although it was not the objective of this study, it could be of importance to bear in mind the potential regulatory influence that $\mathrm{KJ}$ administration could exert on the expression levels of LPC acyltransferase 1 (LPCAT1), contributing to normalise LPC levels to values found in controls. Increased levels of LPCAT1, responsible for the transformation of LPC into $\mathrm{PC}$, have been identified as a promoting factor of hepatoma progression ${ }^{(37)}$, the most severe clinical condition associated with chronic liver inflammation. The decreased PIP2 concentration quantified in $\mathrm{ARa} / \mathrm{KJ}$-fed animals helps to explain the lower proportion of macrophages infiltrated into the liver lobules, suggesting a potential interaction on the Rac2 (Ras-related C3 botulinum toxin substrate 2) signalling pathway.

\section{Conclusions}

The influence of $\mathrm{KJ}$ on several relevant biomarkers affecting ARa-mediated alterations in the liver physiology of hyperglycaemic animals has been monitored. Feeding a prebiotic carbohydrate such as $\mathrm{KJ}(0.5 \%, \mathrm{w} / \mathrm{w}$ diet $)$ to animals administered ARa ameliorated these effects, mainly decreasing plasmatic TAG and ARa concentrations to values similar to those found in controls. Notably, KJ contributed to decreasing the macrophage population infiltrated into the liver and normalised the plasmatic PL profile in hyperglycaemic rats, which could contribute to severity and pathological progression in liver disorders. The results stress the potential relevance that prebiotic carbohydrates could play in ameliorating, but mainly in preventing, liverassociated disorders and adaptive immune responses triggered by tissue injury and failure. Whether these effects could influence the metabolic and inflammatory profile of adipose tissue as well as whole-body insulin sensitivity should be addressed in future research. Further, human trials are warranted to support the clinical relevance and use of prebiotics as coadjutants in nutritional intervention strategies.

\section{Acknowledgements}

M. H. thanks MICINN for his 'Ramón y Cajal' contract.

This work was supported by grants AGL2011-25169, AGL2011-27884 and Consolider Fun-C-Food CSD2007-00063 from the Spanish Ministry of Science and Innovation (MICINN, Spain). M. D.-M. is supported by the High Research Council of the Spanish Government (CSIC) through JAE-Pre Programme, co-funded by European Social Fund (ESF).

Author contributions were as follows: conceived and designed the experiments: J. M. L. and F. J. M.; performed the experiments: J. M. L., M. D.-M., F. J. M. and M. H.; analysed the data and contributed reagents/materials/analysis tools: J. M. L., M. D.-M., F. J. M. and M. H.; wrote the paper: J. M. L., M. D.-M., F. J. M. and M. H.

There are no conflicts of interest to declare.

\section{References}

1. Díez-Municio M, Montilla A, Moreno FJ, et al. (2014) A novel biotechnological process for the efficient synthesis of kojibiose. Green Chem 16, 2219-2226.

2. Nakada T, Nishimoto T, Chaen H, et al. (2003) Chapter 9. In Oligosaccharides in Food and Agriculture 849, 104-117. [G Eggleston and GL Côté, editors]. Washington, DC: American Chemical Society.

3. García-Cayuela T, Díez-Municio M, Herrero M, et al. (2014) Selective fermentation of potential prebiotic lactose-derived oligosaccharides by probiotic bacteria. Int Dairy $J \mathbf{3 8}$, $11-15$.

4. Sanz ML, Gibson GR \& Rastall RA (2005) Influence of disaccharide structure on prebiotic selectivity in vitro. J Agric Food Chem 53, 5192-5199.

5. EFSA Panel on Dietetic Products, Nutrition and Allergies (2014) Scientific opinion on the substantiation of a health claim related to non-digestible carbohydrates and reduction of post-prandial glycaemic responses pursuant to Article 13(5) of Regulation (EC) No 1924/2006. EFSA J 12, 3513.

6. Luo J, Van Yperselle M, Rizkalla SW, et al. (2000) Chronic consumption of short-chain fructooligosaccharides does not affect basal hepatic glucose production or insulin resistance in type 2 diabetics. J Nutr 130, 1572-1577.

7. Parnell JA, Raman M, Rioux KP, et al. (2012) The potential role of prebiotic fibre for treatment and management of nonalcoholic fatty liver disease and associated obesity and insulin resistance. Liver Int 32, 701-711.

8. Pachikian BD, Essaghir A, Demoulin JB, et al. (2013) Prebiotic approach alleviates hepatic steatosis: implication of fatty acid oxidative and cholesterol synthesis pathways. Mol Nutr Food Res 57, 347-359.

9. Brighenti F (2007) Dietary fructans and serum triacylglycerols: a meta-analysis of randomized controlled trials. J Nutr 137, Suppl. 11, 2552S-2556S

10. Roberfroid M, Gibson GR, Hoyles L, et al. (2010) Prebiotic effects: metabolic and health benefits. Br J Nutr 104, Suppl. 2, S1-S63.

11. Laparra JM, Diez-Municio M, Herrero M, et al. (2014) Structural differences of prebiotic oligosaccharides influence their capability to enhance iron absorption in deficient rats. Food Func 5, 2430-2437.

12. Roelofsen H, Priebe MG \& Vonk RJ (2010) The interaction of short-chain fatty acids with adipose tissue: relevance for prevention of type 2 diabetes. Benef Microbes 1, 433-437.

13. Yin J, Peng Y, Wu J, et al. (2014) Toll-like receptor $2 / 4$ links to free fatty acid-induced inflammation and $\beta$-cell dysfunction. J Leukoc Biol 95, 47-52.

14. Ortega-González M, Ocón B, Romero-Calvo I, et al. (2013) Nondigestible oligosaccharides exert nonprebiotic effects on intestinal epithelial cells enhancing the immune response via activation of TLR4-NFkB. Mol Nutr Food Res 58, 384-393.

15. Delzenne NM \& Williams CM (2002) Prebiotics and lipid metabolism. Curr Opin Lipidol 13, 61-67.

16. Barber MN, Risis S, Yang C, et al. (2012) Plasma lysophosphatidylcholine levels are reduced in obesity and type 2 diabetes. PLOS ONE 7, e41456.

17. Fellmann L, Nascimento AR, Tibiriça E, et al. (2013) Murine models for pharmacological studies of the metabolic syndrome. Pharmacol Ther 137, 331-340.

18. Srinivasan K, Viswanad B, Asrat J, et al. (2005) Combination of high fat diet-fed and low-dose streptozotocin-treated rat, a model for type 2 diabetes and pharmacological screening. Pharmacol Res 52, 313-320. 
19. Reed MJ, Meszaros K, Entes LJ, et al. (2000) A new rat model of type 2 diabetes: the fat-fed streptozotocin-treated rat. Metabolism 49, 1390-1394.

20. Laparra JM, Diez-Municio M, Herrero M, et al. (2014) Structural differences of prebiotic oligosaccharides influence their capability to enhance iron absorption in deficient rats. Food Func 5, 2430-2437.

21. Hernández-Hernández O, Marín-Manzano MC, Rubio LA, et al. (2012) Monomer and linkage type of galactooligosaccharides affect their resistance to ileal digestion and prebiotic properties in rats. J Nutr 142, 1232-1239.

22. Ferreiro-Vera FC, Priego-Capote MD \& de Castro L (2012) Comparison of sample preparation approaches for phospholipids profiling in human serum by liquid chromatographytandem mass spectrometry. J Chromatogr A 1240, 21-28.

23. Ginsburg I \& Kohen R (1995) Synergistic effects among oxidants, membrane-damaging agents, fatty acids, proteinases, and xenobiotics: killing of epithelial cells and release of arachidonic acid. Inflammation 19, 101-18.

24. Okamoto Y, Morishita J, Tsuboi K, et al. (2004) Molecular characterization of a phospholipase $\mathrm{D}$ generating anandamide and its congeners. J Biol Chem 279, 5298-5305.

25. Osei-Hyiaman D, DePetrillo M, Pacher P, et al. (2005) Endocannabinoid activation at hepatic CB1 receptors stimulates fatty acid synthesis and contributes to diet-induced obesity. J Clin Invest 115, 1298-1305.

26. Jourdan T, Godlewski G, Cinar R, et al. (2013) Activation of the Nlrp3 inflammasome in infiltrating macrophages by endocannabinoids mediates beta cell loss in type 2 diabetes. Nat Med 19, 1132-1140.

27. Muscolini M, Camperio C, Capuano C, et al. (2013) Phosphatidylinositol 4-phosphate 5-kinase $\alpha$ activation critically contributes to CD28-dependent signaling responses.J Immunol 190, 5279-5286.

28. Locatelli I, Sutti S, Vacchiano M, et al. (2013) NF-кB1 deficiency stimulates the progression of non-alcoholic steatohepatitis
(NASH) in mice by promoting NKT-cell-mediated responses. Clin Sci (Lond) 124, 279-287.

29. Liechtenstein L, Mattijssen F, de Witt NJ, et al. (2010) Angptl4 protects against severe pro-inflammatory effects of dietary saturated fat by inhibiting lipoprotein lipase-dependent uptake of fatty acids in mesenteric lymph node macrophages. Cell Metab 12, 580-592.

30. Zhu P, Goh YY, Chin HF, et al. (2012) Angiopoietin-like 4: a decade of research. Biosci Rep 32, 211-219.

31. Staiger H, Haas C, Machann J, et al. (2009) Muscle-derived angiopoietin-like protein 4 is induced by fatty acids via peroxisome proliferator-activated receptor (PPAR)-delta and is of metabolic relevance in humans. Diabetes $\mathbf{5 8}$, 579-589.

32. Tateya S, Kim F \& Tamori Y (2013) Recent advances in obesity-induced inflammation and insulin resistance. Front Endocrinol (Lausanne) 4, 93

33. Okubo M, Horinishi A, Saito M, et al. (2007) A novel complex deletion-insertion mutation mediated by Alu repetitive elements leads to lipoprotein lipase deficiency. Mol Genet Metab 92, 229-233.

34. Shi H, Kokoeva MV, Inouye K, et al. (2006) TLR4 links innate immunity and fatty acid-induced insulin resistance. $J$ Clin Invest 116, 3015-3025.

35. Tolhurst G, Heffron H, Lam YS, et al. (2012) Short-chain fatty acids stimulate glucagon-like peptide-1 secretion via the G-protein-coupled receptor FFAR2. Diabetes 61, 364-371.

36. Kus V, Flachs P, Kuda O, et al. (2011) Unmasking differential effects of rosiglitazone and pioglitazone in the combination treatment with $n$-3 fatty acids in mice fed a high-fat diet. PLOS ONE 6, e27126.

37. Morita Y, Sakaguchi T, Ikegami K, et al. (2013) Lysophosphatidylcholine acyltransferase 1 altered phospholipid composition and regulated hepatoma progression. I Hepatol 59, 292-299. 